\title{
Fehlstellungen im Unterarmschaftbereich
}

\author{
H.-J. Oestern
}

\section{Zusammenfassung}

Die Osteotomie nach fehlverheilten Unterarmschaftbrüchen ist in Anbetracht der Primärbehandlung mit Plattenosteosynthese sehr selten. Die Indikationsstellung muss eventuelle Bewegungseinschränkungen durch Vernarbungen der Membrana interossea einschließen. Die Unterarmverlängerung hat spezifische Indikationen und Ziele, weist viele Schwierigkeiten auf und ist technisch anspruchsvoll. In den Händen erfahrener Operateure können exzellente Resultate erreicht werden. Die mögliche Komplikationsrate ist anfangs hoch, wird aber mit zunehmender Erfahrung geringer. Der Anteil permanenter Folgen ist gering, wenn Komplikationen früh und suffizient behandelt werden. Die funktionellen und kosmetischen Resultate sind überwiegend gut.

\section{Einleitung}

Die Einschränkung der Rotationsbewegungen des Unterarmes auf Grund einer Fehlstellung, einer zu langen Immobilisierung oder einer Inkongruenz des proximalen oder distalen Radioulnargelenkes war in der Zeit der konservativen Behandlung und frühen intramedullären Osteosyntheseverfahren eine häufige Begleitkomplikation. Auf Grund der exakten Reposition und Retention durch die Plattenosteosynthese tritt diese Spätfolge heute sehr selten auf. Das Problem der Rotationsfehlstellung als Ursache der eingeschränkten Supination/Pronation ist schon seit vielen Jahren bekannt [4]. Auch die Achsenfehlstellung führt zu

OP-JOURNAL 2000; 16: 30-34

(C) Georg Thieme Verlag Stuttgart · New York einer Rotationseinschränkung, insbesondere in den Fällen, in welchen dadurch ein radioulnares Impingement verursacht wird. Dies trifft vor allem für die konservative Frakturbehandlung mit häufig nicht anatomischer Wiederherstellung von Achse und Rotation zu und resultiert in einer erheblichen Bewegungseinschränkung. [5,11,13].

Die Bedeutung der physiologischen Radiuskrümmung auf die Rotationsfähigkeit wurde von den frühen Vertretern der intramedullären Osteosynthese beschrieben [7,10]. Schemitsch und Richards [12] konnten die Bedeutung der Radiuskrümmung auf das funktionelle Resultat für die Plattenosteosynthese nachweisen.

Bereits eine Nichtbeachtung der Radiuskrümmung in einer Größenordnung von 4-5\% führt zu einer Einschränkung der Unterarmrotation von bis zu $20 \%$ und einer Minderung der groben Kraft von über $20 \%$.

Als weitere Ursachen einer Bewegungseinschränkung müssen eine längere Ruhigstellung, Weichteilschaden und ipsilaterale Frakturen mit in die Überlegungen einbezogen werden.

Die Wiederherstellung der Länge und Achse erfolgt im Vergleich zu der unverletzten Gegenseite. Die planerische Zeichnung muss die exakte Kongruenz im distalen und proximalen Radioulnargelenk bedenken. Die Achsenfehlstellung wird durch Einfalzen von kortikospongiösen Blöcken, die Retention durch eine Plattenosteosynthese erreicht.

Die Korrekturosteotomie beim Erwachsenen nach Unterarmschaftfraktur stellt in Anbetracht der adäquaten Primärbehandlung heute eine absolute Rarität dar.

\section{Funktionelle Anatomie} und Biomechanik

Knöchernes Skelett, muskuläre Verbindungen und die Membrana interossea gewähren in engem Zusammenspiel die Feinmotorik in Ellenbogen- und Handgelenk.

Durch die Krümmung des Radius von jeweils $10^{\circ}$ in beiden Ebenen ist seine Rotation um die Ulna möglich. Der Hauptdruckaufnehmer ist die Ulna, die dementsprechend proximal breit und distal schmal ausgebildet ist. Der Radius ist umgekehrt aufgebaut. Die Markhöhlen von Radius und Ulna sind im mittleren Segment besonders eng. Die beiden Unterarmknochen sind von proximal nach distal durch Muskeln verbunden: M. pronator teres, M. supinator und den M. pronator quadratus.

Die Membrana interossea antebrachii beginnt etwa $6 \mathrm{~cm}$ distal des Radiusköpfchens und verläuft bis zum distalen Radioulnargelenk. Das proximale und distale Viertel der Membran ist dünn und durchscheinend, während der zentrale Bereich durch viele kollagene Bandstrukturen dicker ist. Diese Fasern haben einen schrägen Verlauf von etwa $20^{\circ}$ vom proximalen Radius zur distalen Ulna und machen etwa 40-60\% der Membranfläche aus. Sie entspringen am Radius 7 $9 \mathrm{~cm}$ unterhalb des Radiusköpfchens und inserieren an der Ulna zwischen 10,5 und $16,6 \mathrm{~cm}$ unterhalb des Olekranons.

Nur bei äußerster Pronation erschlafft die Membrana interossea in allen Fasern. Ansonsten sind stets Faserzüge gespannt, die eine Parallelverschiebung der beiden Unterarmknochen verhindern und die beiden Radioulnargelenke gegen Längsverschiebung sichern. Über die Membrana interossea verläuft ein Lasttransfer von proximal nach distal von der Ulna auf den Radius. 


\section{Defekte und Entwicklungsstörungen am Unterarm}

An der oberen Extremität führen angeborene Defektbildungen und Entwicklungsstörungen häufig zu komplexen Deformitäten mit Verkürzung und Achsenfehlstellungen [1]. Aufgrund der geringeren mechanischen Belastung werden diese Deformitäten besser toleriert und haben eine geringere funktionelle Bedeutung als an der unteren Extremität. Deshalb sind im Gegensatz zur unteren Extremität die Korrekturen an der oberen Extremität weniger häufig.

\section{Klassifikation}

Villa etal. [15] beschrieben 5 Typen unter den Längendeformitäten des Unterarmes. Diese Klassifikation basiert im wesentlichen auf der Ätiologie:

Typ I: Alleinige Verkürzung des Radius

Typ II: Alleinige Verkürzung der Ulna

A: Ohne Luxation des Radiusköpfchens

B: Mit Luxation des Radiusköpfchens

Typ III: Verkürzung der Ulna, der Radius ist nicht angelegt

Typ IV: Proportionale Verkürzung beider Knochen

Typ V: Disproportionale Verkürzung beider Knochen

Daraus ergibt sich das folgende therapeutische Verfahren:

Typ I: Verlängerung nur des Radius

Typ II A:Verlängerung nur der Ulna

Typ II B: Verlängerung der Ulna mit distalem Transport des Radius und Einrichtung des Radiusköpfchens

Typ III: Verlängerung der Ulna

Typ IV: Verlängerung beider Unterarmknochen in gleicher Länge

Typ V: Verlängerung beider Unterarmknochen mit unterschiedlicher Ausdehnung

\section{Operative Verfahren}

Die monolaterale externe Fixation erscheint bei der Unterarmverlängerung technisch einfacher. Auf der anderen Seite ist die monolaterale Fixierung für komplexe Korrekturen weniger gut anwendbar. Weiterhin besteht eine Einschränkung hinsichtlich der Zahl und der Lokalisation der Osteotomien und im Hinblick auf die Fixierungsmöglichkeiten. Die monolaterale Montage ist am geeignetsten für diaphysäre Verlängerungen ohne begleitende Deformitäten. Die Knochenbildung ist diaphysär weniger ausgeprägt als metaphysär und der neugebildete Knochen ist bei der diaphysären Verlängerung aufgrund der geringeren Querschnittsfläche bis zum vollständigen Remodelling relativ schwach. Die monolaterale Fixierung ist an der Ulna einfacher anzuwenden als am Radius, weil die Ulna ein gerader subkutaner Knochen ist und proximal eine relativ große Querschnittsfläche aufweist. Die Fixierung der Hand wird empfohlen bei einer Verlängerung um mehr als $3 \mathrm{~cm}$ am Radius und bei alleiniger Ulna-Anlage. Kontrakturen sowie der Verlust der Handgelenks- und Fingerbeweglichkeit können so vermieden werden. Die transartikuläre Fixierung ist bei monolateraler Montage schwierig. Bei Ulnaverlängerung ist die Transfixation des Handgelenkes nicht so entscheidend, nur der Radius muss proximal transfixiert werden, um einen Transport des Radiusköpfchens nach distal zu vermeiden. Bei monolateraler Montage ist es schwierig, den Radius in dieser Position zu fixieren, dies gelingt einfacher mit einem zirkulären Fixateur. Der Ilizarov-Apparat kann mit Drähten, Schanzschen Schrauben oder kombiniert zur Anwendung kommen.

\section{Fehlstellungen durch Wachstumsstillstand}

Wachstumsstillstand kann posttraumatisch, kongenital oder durch Entwicklungsstörungen hervorgerufen werden.

Ein partieller peripherer Wachstumsstillstand des Radius führt zu einer progredienten Deformität und Längendifferenz im Vergleich zur Ulna.

Ein kompletter Wachstumsstillstand resultiert nicht nur in einer Längendifferenz, sondern auch in einer zunehmenden Sprengung des distalen Radioulnargelenkes.

Die Deformität durch Wachstumsstillstand hängt von dem exakten Ort der Verknöcherung der Epiphysenfuge ab. Knöcherne Fugenbrücken auf der Radialseite führen zu einer radialen Deviation, dorsale Verknöcherungen zu einer Dorsalabkippung und knöcherne Brücken zwischen der Frontal- und Sagittalebene zu schräg verlaufenden Achsabweichungen. Die Fehlstellung stellt sich auf der Schrägaufnahme größer dar als im a.-p. oder seitlichen Röntgenbild. Die Größe und der Verlauf der tatsächlichen Fehlstellungsebene können trigonometrisch, einfacher grafisch berechnet werden.

\section{Berechnung der Fehlstellung}

Die grafische Methode ist unkompliziert und exakt und kann mit einfachen Mitteln durchgeführt werden. Sie stellt eine Annährung an die trigonometrische Berechnung dar und ist für Winkel unter $45^{\circ}$ verhältnismäßig genau.

\section{Grafische Methode}

Mit der grafischen Methode werden eine $\mathrm{X}$ - und Y-Achse aufgezeichnet.

$1 \mathrm{~mm}$ entspricht $1^{\circ}$. Die X-Achse stellt die Frontalebene und die Y-Achse die Sagittalebene dar. Die Achsen entsprechen der Neutral-0-Methode. Die Fehlstellung wird in der a.-p. Ebene und im seitlichen Röntgenbild gemessen, die Messung im a.p.-Bild wird auf der X-Achse und die laterale auf der Y-Achse dargestellt. Diese Punkte werden in der Richtung der Winkelspitze in dieser Ebene markiert. Deshalb wird eine $38^{\circ}$-radial-Abwinklung $38 \mathrm{~mm}$ ulnaseitig der X-Achse aufgetragen. Ähnlich bewirkt eine $42^{\circ}$-Dorsalabwinklung (Spitze palmar), dass der Punkt $42 \mathrm{~mm}$ auf der Palmarseite der Y-Achse markiert wird. Wird nun eine Verbindungslinie zum Schnittpunkt gemessen, stellt dieser Wert den Fehlstellungswinkel dar (Abb. 1).

\section{Operative Verfahren}

Beim kompletten Wachstumsstillstand ohne Deformität besteht das Ziel der Behandlung in der Beseitigung des ulnokarpalen Impingements sowie in der Verbesserung der Kraft und des Bewegungsausmaßes. Bei Längenunterschieden unter $1 \mathrm{~cm}$ und jenseits der Wachstumsphase ist die Ulnaverkürzungsosteotomie die einfachste Methode.

Bei größeren Fehlstellungen und bei Kindern mit einem zu erwartenden beträchtlichen Wachstum muss eine Verlängerung des Radius eingeplant werden.

Da die Ulna nach der Verlängerung des Radius weiter wächst, resultiert ein erneuter Längenunterschied.

Drei Möglichkeiten stehen zur Verfügung [6]:

1. die Resektion der Verknöcherung

2. die sekundäre Verlängerung nach Wachstumsschluss

3. die Überkorrektur unter Berechnung des voraussichtlichen Wachstumsunterschiedes bei Fugenschluss 


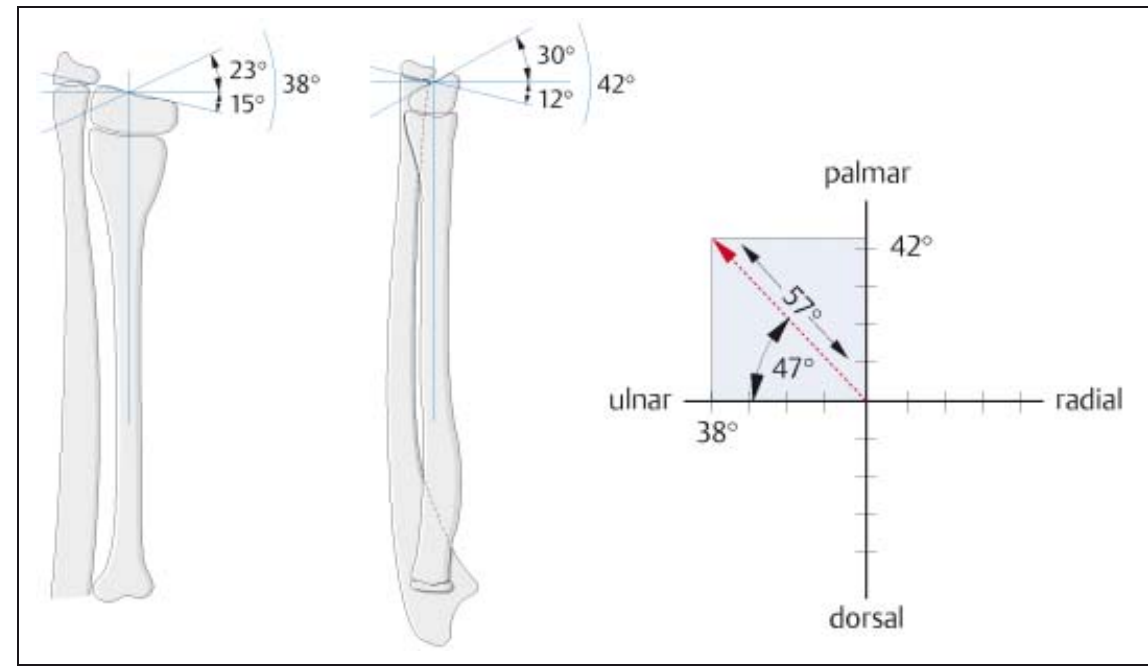

Abb.1 Die Fehlstellung beträgt im a.p. Bild $38^{\circ}$ und lateral $42^{\circ}$. Die a.p Fehlstellung ist auf der $X$-Achse und die laterale Fehlstellung auf der Y-Achse markiert $-1 \mathrm{~mm}$ entspricht $1^{\circ}$. Die Länge vom Kreuzungspunkt bis zum Schnittpunkt der jeweiligen Senkrechten zum Winkel von $38^{\circ}$ und $42^{\circ}$ entspricht $57 \mathrm{~mm}$, entsprechend $57^{\circ}$. Dies ist der Fehlstellungswinkel in der Schrägaufnahme. Die Richtung der Fehlstellungsebene zur Frontalebene (X-Achse) beträgt $47^{\circ}$. Die Richtung des Krümmungsscheitels der Fehlstellung ist ulnopalmar (aus: Buck-Gramcko, D.: Congenital Malformations of the Hand and Forearm).

Die Resektion der Verknöcherung sollte bis zum 10. Lebensjahr beim partiellen zentralen Wachstumsstillstand und bei weniger als $50 \%$ überbrückter Wachstumszone durchgeführt werden.

Wiederholte Verlängerungsoperationen sind eine gute Indikation für größere zu erwartende Wachstumsunterschiede, wenn eine Resektion nicht durchgeführt werden kann. Zwei kleinere Verlängerungen werden besser toleriert als eine risikoreiche größere Verlängerungsoperation. Überkorrekturen werden bei den Kindern durchgeführt, deren erwarteter Längenunterschied bei Wachstumsabschluss maximal $3 \mathrm{~cm}$ beträgt. Die Wachstumsprognose wird nach den Tabellen von Pritchett [9] durchgeführt. Überkorrekturen drängen das Ulnaköpfchen aus dem distalen Radioulnargelenk und wirken deshalb wie ein Operationsverfahren nach Darrach [3]. Die Ulna wächst weiter und kann die Länge des überkorrigierten Radius erreichen. Durch diese langsame Wachstumsangleichung kann sich die Ulna dem distalen Radioulnargelenk anpassen. Erreicht die Ulna das Gelenk, kann eine Rotationseinbuße eintreten. Um eine zu starke Überkorrektur zu vermeiden, kann auch eine geringere Überkorrektur mit distaler Ulnaepiphysiodese kombiniert werden. Die Epiphysiodese wird dann durchgeführt, wenn die Ulna den Radius erreicht hat.

\section{Operative Technik}

Ein Ring-Fixateur mit zwei parallelen Ringen wird vorgefertigt. Ein distaler Radiusreferenzdraht wird palmar radial zur A. radialis und in dorsoulnarer Richtung radial des distalen Radioulnargelenkes eingebracht. Dieser Draht läuft rechtwinklig zur Längsachse der Ulna. Der Fixateur wird auf den Unterarm zentriert und am distalen Draht befestigt. Ein proximaler Draht oder eine Schanzsche Schraube wird in Unterarmmitte eingebracht. Wird ein Draht gewählt, wird er von palmar nach dorsal so weit radial wie möglich eingebracht. Bei diesem Draht ist der N. superficialis des N. radialis gefährdet. Deshalb ist eine Schanzsche Schraube, die von der subkutanen Grenze des Radius eingebracht wird, weniger gefährdend. Der Draht oder die Schraube wird an dem proximalen Ring fixiert. Die Fixation wird komplettiert durch Einbringen einer weiteren Schanzschen Schraube an jeden Ring. Der distale Draht wird in das Tuberkulum listeri im rechten Winkel zum Draht eingebracht. Der proximale Pin wird distal des 1 . Pin von der dorsoradialen Seite des Unterarmes eingeführt [6].

Die radiale Osteotomie liegt an der distalen Metaphyse proximal der distalen Fixierung. Über eine 5-10 mm longitudinale Inzision erfolgt an der Radialseite die subperiostale Freilegung des distalen
Radius. Multiple Bohrungen werden mit einem 3,2-mm-Bohrer durchgeführt und mit einem feinen Osteotom wird die Osteotomie durch Drehung des Osteotoms um $90^{\circ}$ komplettiert. Werden mehr als $2-3 \mathrm{~cm}$ verlängert, sollte auch die Hand in den Ringfixateur eingeschlossen werden, z. B. durch einen Metakarpaldraht.

\section{Nachbehandlung}

Die Distraktion beginnt 3-7 Tage nach Osteotomie abhängig vom Alter des Patienten. Die Distraktionsgeschwindigkeit beträgt $0,25 \mathrm{~mm}, 4 \times \mathrm{am}$ Tag. Die Knochenneubildung ist gewöhnlich im distalen Radius aufgrund des großen metaphysären Querschnitts gut. Wird die Hand nicht an den Rahmen durch einen Metakarpaldraht fixiert, sollte das Handgelenk zumindest durch eine Schiene in der Funktionsposition gehalten werden, um so eine Flexionskontraktur zu vermeiden. Eine intensive physikalische Therapie ist postoperativ dringend erforderlich.

\section{Fehlstellungen durch multiple kartilaginäre Exostosen}

Multiple kartilaginäre Exostosen stellen eine autosomale Erkrankung dar, die entweder familiär oder sekundär aufgrund einer spontanen Mutation entsteht.

Kartilaginäre Exostosen können dann zu Problemen führen, wenn 2 parallele Knochen wie am Unterschenkel und am Unterarm vorhanden sind. Am Unterarm sind die distale Ulna und ihre Epiphyse häufig von kartilaginären Exostosen betroffen, die zu einer relativen Verkürzung und Deviation der Ulna führen. Die unterschiedliche Wachstumsrate kann auch zu einer Luxation des Radiusköpfchens führen. Die Radiusköpfchenverrenkung kann ebenfalls als Folge einer proximalen interossär gelegenen kartilaginären Exostose auftreten. Dies sollte computertomographisch verifiziert werden. Eine Valgusdeformität auch ohne lokale kartilaginäre Exostose kann sekundär durch laterale Druckeinwirkung des Humerus bei Sub- oder Luxation des Radiusköpfchens entstehen. Eine Valgusposition des Radiushalses macht die Reposition des Radiusköpfchens besonders schwierig.

Distal gelegene kartilaginäre Exostosen sind häufig und können zu einer Deformität des distalen Radius führen. Interossäre kartilaginäre Exostosen von Radius und Ulna schränken die Unterarmdre- 
hung ein, auch wenn keine Fehlstellung des anderen Knochens besteht. Außer einer Verkürzung der Ulna ist auch der Unterarm häufig insgesamt bis zu $5 \mathrm{~cm}$ kürzer als die normale Gegenseite.

Für die Therapieplanung müssen zum einen die Fehlstellungen und zum anderen die Folgen kartilaginärer Exostosen aufgelistet werden:

1. Fehlstellungen mit multiplen kartilaginären Exostosen: interossäre kartilaginäre Exostosen Ulna kürzer als Radius vermehrte Ulnaabweichung des distalen Radius

Subluxation oder Verrenkung des Radiusköpfchens

Valgisation des Radiushalses

Radius kürzer als die Gegenseite

2. Probleme mit multiplen kartilaginären Exostosen:

Einschränkung der Unterarmdrehung Ulnadeviation der Hand

Schmerzhafte Schwellung im lateralen Bereich des Ellenbogens

Kurzer Unterarm

Die Behandlung muss alle oben genannten Deformitäten mit einschließen und kann in Abhängigkeit von der Schwere der klinischen Symptomatik in 4 Bereiche aufgeteilt werden [6].

Die erste operative Intervention ist die Resektion der interossären kartilaginären Exostosen, die die Unterarmdrehung einschränken. Sind sogenannte „Kissing Osteochondrome“ vorhanden, vereinfacht die Resektion die Verlängerung des betroffenen Knochens. Osteotomien und Verlängerungen können zur selben Zeit wie die Exostosenresektion durchgeführt werden.

Ein schrittweises operatives Vorgehen vermindert das Risiko für Brückenkallus und verbessert die Unterarmdrehung.

Eine zweite Behandlungsindikation umfaßt die Ulnadeviation des Handgelenkes, die auf die fehlende Ulna-Beteiligung am Handgelenk und die vermehrte Ulnaneigung des distalen Radius zurückzuführen ist. Beide Fehlstellungen können simultan durch eine distale Radiuskorrekturosteotomie mit Ulnaverlängerung behoben werden.

Die 3. Behandlungsindikation ist die Subluxation oder Verrenkung des Radiushalses. Diese Radiusverrenkung ist symptomatisch, da sie in zunehmendem Alter erfolgt. Das Radiusköpfchen muss durch eine Varisationsosteotomie des Radiushalses reponiert werden.

Eine Radiusköpfchenverrenkung findet sich immer in Begleitung mit einer zu kurzen Ulna.

Deshalb sollten beide Fehlstellungen operativ angegangen werden. Die Ulna sollte verlängert werden, um den Längenunterschied im Bereich des Handgelenkes auszugleichen und der Radiushals sollte osteotomiert werden, um das Radiusköpfchen zu reponieren.

Am besten geschieht dies in 2 Schritten:

Der 1. Schritt umfasst die Verlängerung der Ulna, der 2. Schritt die Reposition des Radiusköpfchens mit Osteotomie des Radiushalses, um den Radius an die Ulna proximal zu fixieren.

Die 4. Behandlungsüberlegung ist die Verlängerung, um die Unterarmlänge auszugleichen. Multiple kartilaginäre Exostosen können beide Unterarme in einem unterschiedlichen Ausmaß beeinflussen. Zum Zeitpunkt der Skelettreifung wird die stärker betroffene Seite $3-5 \mathrm{~cm}$ kürzer als die andere Seite. Die entsprechende Verlängerung der Unterarme um diesen Betrag bedeutet keine funktionelle, wohl aber eine ästhetische Verbesserung. Ist die Unterarmbewegung eingeschränkt, ist die Verlängerung in jedem Fall gerechtfertigt. Je normaler die Unterarmdrehung, desto größer ist das Risiko eines Bewegungsverlustes. Bei Patienten mit multiplen kartilaginären Exostosen ist die Unterarmrotationsbewegung bereits eingeschränkt. Deshalb ist eine Verlängerung um mehr als den Betrag, der zur Fehlstellungskorrektur benötigt wird, nicht ratsam.

\section{Komplikationen der Unterarmverlängerung}

\section{Pintrakt-Infektion}

Pintrakt-Infektionen sind distal weniger häufig als in Unterarmmitte und proximal.

\section{Nerven- und Gefäßverletzungen}

Neurovaskuläre Verletzungen können durch den Pin oder die Distraktion hervorgerufen werden. Durch Pin verursachte neurovaskuläre Verletzungen gehen gewöhnlich auf Transfixationsdrähte zurück. Am Unterarm existieren in Anbetracht der vielen wichtigen motorischen, sensorischen und vaskulären Strukturen nur wenige absolut sichere anatomische Schichten für die Transfixationsdrähte. Deshalb wird die Halfpin-Fixation empfohlen. Während der Drahtinsertion sollte anästhesiologisch keine neuromuskuläre Blockierung erfolgen. Berührt der Draht oder Bohrer einen Nerv, reagieren die Finger und die Richtung muss geändert werden. Wird die gegenüberliegende Kortikalis durchbohrt, wird der Bohrvorgang beendet und der Draht stumpf durch die Weichteile geschoben, unter Beachtung einer nerval ausgelösten Fingerbewegung.

Der tiefe und der sensorische Ast des N. radialis sind am meisten gefährdet: Der sensorische Ast von Unterarmmitte nach distal, der tiefe $\mathrm{N}$. radialis-Ast im proximalen Bereich. Paley [6] beobachtete 3 Nervus-radialis-Läsionen, 2 Nervusmedianus-Läsionen, die alle rückläufig waren. Wird eine Nervenverletzung diagnostiziert, muss der Draht sofort entfernt werden. Die Dekompression des Nerven ist indiziert, wenn exakt der Ort der Schädigung feststeht und die Schwellung unter Umständen einen weiteren Druck auf den Nerven ausübt, z. B. N. medianus im Karpaltunnel oder N. radialis am Radiushals oder N. ulnaris beim Eintritt in die Guyon-Loge. Distraktionsbezogene Nervenverletzungen sind selten und gewöhnlich auffällig durch sensorische Symptome. Eine genaue Diagnostik von Hyper-, Hypoästhesien oder Schmerzsymptomen kann derartige Verletzungen vermeiden.

Gefäßverletzungen durch einen Draht sind ebenso selten wie Kompartmentsyndrome durch perkutane Osteotomien.

\section{Muskelkontrakturen}

Unterarmverlängerungen neigen $\mathrm{zu}$ Ellbogen-, Finger- und Handgelenksflexionskontrakturen. Da Muskeln beteiligt sind, die mehr als ein Gelenk kreuzen, muss der Muskel physikalisch aufgedehnt werden. Um dies zu erleichtern, sollte das Handgelenk entweder durch einen Metakarpaldraht oder durch eine palmar angelegte Orthese in einer Neutralposition oder leichten Extension geschient werden. Nachtschienen für Ellbogengelenk und Finger sind ebenso geeignet. Am Ellbogen wird vorzugsweise eine dynamische Schiene angelegt, wohingegen an den Fingern eine statische Orthese oder elastische Schienen Anwendung finden. Bei einem Extensionsdefizit über $30^{\circ}$ im Fingerbereich, sollte die Verlängerung 
unterbrochen werden, bis sich die Fingerstreckung normalisiert hat. Eine permanente Kontraktur der Metakarpophalangealgelenke kann bei Patienten mit verminderter Compliance auftreten oder wenn auf Kosten der Funktion eine Verlängerung erreicht wird.

Die Handgelenksbeweglichkeit normalisiert sich gewöhnlich nach Entfernung des Ringfixateurs wieder, dies kann aber bis zu 2 Jahre dauern.

Supinations- und Pronationsbewegungen sind gegenüber einem Dauerschaden am empfindlichsten. Patienten mit einer vorbestehenden eingeschränkten Unterarmdrehung verlieren gewöhnlich keine weitere Rotation. Bei Patienten mit normaler Unterarmdrehung besteht das Risiko, Supination oder Pronation oder beides einzubüßen. Das Verhältnis von Radius und Ulna muss nach Distraktion wieder normalisiert sein, um eine mechanische Blockade zwischen Radius und Ulna zu vermeiden. Besonders wichtig ist dies in den Fällen mit solitärer Verkürzung des Radius, die durch eine isolierte Verlängerung des Radius behandelt wird.

Bei einer Verlängerung von Radius und Ulna besteht das höchste Risiko eines Rotationsverlustes.

Ein Rotationsverlust kann ebenfalls durch eine Synostose eintreten, die durch die Transfixationsdrähte entsteht. Um dies zu verhindern, sollte die Ulna- und Radiusosteotomie an verschiedenen Höhen erfolgen.

\section{Ossäre Probleme}

Eine vorzeitige Konsolidierung ist im Gegensatz zu einem verzögerten Durchbau im Vorderarmbereich eher ungewöhnlich. Die Kortikalisdurchbauung benötigt häufig einen langen Zeitraum. Nach Entfernung der Verlängerungsmontage sollte deshalb der Unterarm noch im Gipsverband geschützt werden. Eine Refraktur ist ungewöhnlich bei Verlängerungen eines Knochens. Der intakte Knochen scheint den verlängerten Knochen zu schienen.

\section{Ergebnisse}

Villa [15] berichtete über Verlängerungsoperationen zwischen 2 und $13 \mathrm{~cm}$, entsprechend $10-143 \%$ bei 12 Patienten. Die Konsolidierung des Knochens wurde nach 3-19 Monaten ohne zusätzliche Knochentransplantationen erreicht. 11 der 12 Patienten zeigten funktionelle und kosmetische Verbesserungen, Komplikationen bestanden in drei temporären tiefen $\mathrm{N}$. radialis-Paresen, zwei Pseudarthrosen, drei verzögerten Heilungen und zwei Refrakturen.

Tetsworth [14] berichtete über 13 Unterarmverlängerungen, die alle durch einen Chirurgen versorgt wurden. Insgesamt wurde $8 \times$ am Radius und $9 \times$ an der Ulna verlängert. Die Verlängerungen der Ulna betrugen $3,4-11,7 \mathrm{~cm}$ und die Behandlungszeit dauerte im Durchschnitt 6,1 Monate (3,4-9,3 Monate). Die Verlängerung des Radius umfasste $1,7-6,2 \mathrm{~cm}$ und die Behandlungszeit dauerte im Durchschnitt 5 Monate. Bei 11 der $13 \mathrm{~Pa}-$ tienten wurde gleichzeitig eine Korrektur der Fehlstellung durchgeführt. Unter den 10 Komplikationen fanden sich zwei oberflächliche Pintrakt-Infektionen, eine vorzeitige Konsolidierung, die eine Rekortikotomie erforderlich machte sowie zwei Fehlstellungen des Regenerats nach Fixateurentfernung.

Des Weiteren wurden zwei temporäre N. radialis-Paresen und eine Reflexdystrophie beobachtet. Insgesamt waren die funktionellen Resultate sehr gut.

Pritchett [8] verlängerte 10 Ulnae bei 8 Patienten mit hereditären multiplen Exostosen. Bei 3 Patienten führte er eine akute Verlängerung von 1,2$2,5 \mathrm{~cm}$ mit sofortiger Knochentransplantation und interner Fixierung durch. Bei den anderen 5 Patienten erfolgte eine sofortige Diastase von $1 \mathrm{~cm}$, gefolgt durch eine graduelle Distraktion 2,3-4 cm mit Fixateur externe.

Burgess [2] beschrieb einen Fall einer Radiusverlängerung bei einem 10-jährigen Jungen mit einer Pseudarthrose des Radius sekundär nach Infekt mit Wachstumsstillstand. Mit der Ilizarov-Methode komprimierte er die distale Pseudarthrose und verlängerte den Radius um 2,4 cm durch eine proximale Kortikotomie.

\section{Literatur}

${ }^{1}$ Buck-Gramcko D. Congenital Malformations of the Hand and Forearm. Churchill Livingstone - London Edinburgh Philadelphia Sydney Toronto 1998

${ }^{2}$ Burgess, RC. Use of the Ilizarov technique to treat radial non-union with physeal arrest. J Hand Surg 1991; 16A: 928-931

${ }^{3}$ Darrach W. Partial excision of the lower shaft of the ulnar for deformity following Colles' fracture. Ann Surg 1913; 57: 764-765

${ }^{4}$ Evans EM. Rotational deformities in the treatment of fractures of both bones of the forearm. J. Bone Joint Surg., 1945; 27: $373-379$

${ }^{5}$ Matthews LS, Kaufer H, Garver DF, Sonstegard DA. The effect on supination-pronation of angular malalignment of fractures of both bones of the forearm. An experimental study. J. Bone Joint Surg. 1982; 64 A: 14-17

${ }^{6}$ Paley D, Herzenberg JE. Distraction Treatment of the Forearm In: Congenital Malformations of the Hand and Forearm. BuckGramcko, D. Churchill Livingstone - London Edinburgh Philadelphia Sydney Toronto 1998; $73-117$

${ }^{7}$ Patrick J. A study of supination and pronation, with especial reference to the treatment of forearm fractures. J. Bone Joint Surg. 1946; 28B: $737-748$

${ }^{8}$ Pritchett JW. Lengthening the ulna in patients with hereditary multiple exostoses. J Bone Joint Surg 1986; 68B: $561-565$

${ }^{9}$ Pritchett JW. Growth and predictions of growth in the upper extremity. J Bone Joint Surg 1988; 70A: 520-524

${ }^{10}$ Sage FP. Medullary fixation of fractures of the forearm: a study of the medullary canal of the radius and a report on 50 fractures of the radius treated with a pre-bent triangular nail. J. Bone Joint Surg. 1982; 64A: 857-863

${ }^{11}$ Sarmiento A, Cooper JS, Sinclair WF. Forearm fractures. Early functional bracing - a preliminary report. J. Bone Joint Surg. 1975; 51 A: 297-304

${ }^{12}$ Schemitsch EH, Richards RH. The effect of malunion on functional outcome after plate fixation of fractures of both bones of the forearm in adults. J. Bone Joint Surg. 1992; 74A: $1068-1078$

${ }^{13}$ Tarr RR, Garfinkel AI, Sarmiento A. The effects of angular and rotational deformities of both bones of the forearm. J. Bone Joint Surg. 1984; 66A: 65-70

${ }^{14}$ Tetsworth K, Krome J, Paley D. Lengthening and deformity correction of the upper extremity by the Ilizarov technique. Orthop clin North Am 1990; 22: 689-713

15 Villa A, Paley D, Catagni MA, et al. Lengthening of the forearm by the Ilizarov technique. Clin. Orthop 1990; 250: 125-137

\section{Prof. Dr. med. H.-J. Oestern}

Chefarzt der Klinik

für Unfall- und Wiederher-

stellungschirurgie

Allgemeines Krankenhaus

Siemensplatz 4

29223 Celle 